\title{
A Decision Support Tool for Agricultural Applications Based on Computational Social Choice and Argumentation
}

Nikos Karanikolas, Department of Computer Engineering and Informatics, University of Patras, Patras, Greece Pierre Bisquert, IATE, University Montpellier, INRA, CIRAD, Montpellier SupAgro, Montpellier, France \& INRIA GraphIK, LIRMM, Montpellier, France

Patrice Buche, IATE, University Montpellier, INRA, CIRAD, Montpellier SupAgro, Montpellier, France \& INRIA GraphIK, LIRMM, Montpellier, France

Christos Kaklamanis, Department of Computer Engineering and Informatics, University of Patras, Patras, Greece \& CTI Computer Technology Institute and Press "Diophantus", Patras, Greece

Rallou Thomopoulos, IATE, University Montpellier, INRA, CIRAD, Montpellier SupAgro, Montpellier, France \& INRIA GraphIK, LIRMM, Montpellier, France

\begin{abstract}
In the current article, the authors describe an applied procedure to support collective decision making for applications in agriculture. An extended 2-page abstract of this paper has been accepted by the EFITA WCCA congress and this manuscript is an extended version of this submission. The problem the authors are facing in this paper is how to reach the best decision regarding issues coming from agricultural engineering with the aid of Computational Social Choice (CSC) and Argumentation Framework (AF). In the literature of decision-making, several approaches from the domains of CSC and AF have been used autonomously to support decisions. It is our belief that with the combination of these two fields the authors can propose socially fair decisions which take into account both (1) the involved agents' preferences and (2) the justifications behind these preferences. Therefore, this article implements a software tool for decision-making which is composed of two main systems, i.e., the social choice system and the deliberation system. In this article, the authors describe thoroughly the social choice system of our tool and how it can be applied to different alternatives on the valorization of materials coming from agriculture. As an example, that is demonstrated an application of our tool in the context of Ecobiocap European project where several decision problems are to be addressed. These decision problems consist in finding the best solutions for questions regarding food packaging and end-of-life management.
\end{abstract}

\section{KEYWORDS}

Agriculture, Argumentation Framework, Computational Social Choice, Decision-Making 


\section{INTRODUCTION}

Collective decision-making and preference aggregation are widely used in modern societies. The most well-known example of collective decision-making is political elections but it is not the only one since there are a number of situations where the aggregation of the individual preferences is needed to take a decision. Consider for example situations such as a group of friends choosing where to have dinner or a committee board choosing what is the best strategy for the company. In all these cases what we are seeking is a way to fairly aggregate the preferences of the individual agents into a collective preference and thus obtain a decision which satisfies the group as a total. This setting can be directly applied also in the decision-making for agricultural problems. Such an example is a problem where the decision lays in evaluating the interest and potential of marketing new generation packaging made of agro-waste materials. Here, the consumers are asked to express preferences among different packaging.

We are studying the classical collective decision-making problem, where we have a set of alternative options and a set of agents and each agent is called to express her preference over the alternatives by producing a linear order on them. In most of these collective decision-making problems the preference aggregation is done by using simple aggregation methods, such as the plurality voting rule, and the tools that are used are simple and not even intended to serve this purpose. For example, doodle is one such unsuitable tool that is used for preference aggregation while its original functionality was for scheduling joint activities. Therefore, we propose a procedure for supporting more complex collective decision-making problems, which can be directly applied in the context of agricultural applications. Our objective is to expand this classical collective decision-making problem by asking the agents to consider the reasoning behind the linear ordering of the alternatives. Hence, the goal of this work is to build, i.e., design and implement, a software tool for decision-making that takes into account theoretical insights from social choice and argumentation in order to propose social fair decisions that take into account the preferences of the agents and the reasoning behind these preferences.

\subsection{Related Work}

The application of Argumentation Framework and Social Choice having as a goal the support of Decision Analysis is a relatively new field; however there has been significant research towards decision-making on both of these fields independently.

Multi-Criteria Decision Analysis (MCDA) has first started evolving in the 60s when Benayoun, Roy, and Sussman $(1966,1968)$ introduced the class of ELECTRE methods for aggregating preferences expressed on multiple criteria. The ELECTRE methods set the foundations for the "outranking" methods (Ostanello, 1985; Roy, 1991) which was the first step of integrating notions of social choice into decision-making. MCDA and CSC are two closely correlated fields whose objective is to aggregate the partial preferences into a collective preference. Arrow and Raynaud (1986) were the first that presented a general exploration of the links between CSC and MCDA. Voting methods from social choice theory have been integrated in the analysis of some popular aggregation methods in multi-criteria decision-aiding. Let us mention, for example, the Condorcet method, on which the ordinal methods in multi criteria analysis, e.g., (Roy, 1991; Roy \& Bouyssou, 1993) are based and the Borda method on which the cardinal ones, e.g., (Von Winterfeldt \& Edwards, 1986; Keeney \& Raiffa, 1993) are based.

Several researchers have proposed the use of Argumentation in decision-making analysis. The work of Fox and Parsons (1997) is one of the first works that tried to deploy an argumentative approach to decision-making stating the difference between argumentation for actions and argumentation for beliefs. The objective of most of the argumentation-based approaches (Karacapilidis \& Papadias, 1998; Morge \& Mancarella, 2007) is to select the best solution (alternative option) compared to decision analysis, where the objective can have several different problem statements, i.e., choosing, 
rejecting, ranking, classifying the set of alternatives. Regarding the aggregation, several approaches (Amgoud et al., 2005; Bonnefon \& Fargier, 2006) used procedures based only on the number or the strength of arguments, while in decision analysis many aggregation procedures have been proposed. Another seminal work towards the usage of argumentation frameworks in decision-making is the one by (Amgoud \& Prade, 2009) which proposes an abstract argumentation-based framework for decisionmaking. The model follows a 2-step procedure where at first the arguments for beliefs and options are built and at the second step we have pairwise comparisons of the options using decision principles. Also, our team adopted argumentation framework in decision-making regarding applications from the agricultural domain (Tamani et al., 2015) and (Yun et al., 2016).

Recently, significant research on the applicative side of CSC has been deployed. For instance, some web-based tools for preference aggregation that allow users to create their own polls and conduct voting have been created. The objective of these web applications is to help collective decision-making by facilitating the voting's procedure for the non-experts. Among them, the three most indicative ones are Pnyx (Brandt et al., 2015), Robovote (Merlob et al., 2016), and Whale (Bouveret, 2010). Observe that these poll tools are purely social choice oriented and are used for obtaining a collective decision in a single poll. In contrast, our tool is a decision-making tool that allows to derive decisions, based on preferences and justifications, for multiple decision problems.

\subsection{Our Work}

Our results include the design and the implementation of a decision-making tool, that will be used for agricultural material valorization. Our goal is to design a sophisticated tool that is adapted to the needs of surveys conducted for agricultural problems and produces decisions based on the justified preferences of the agents. Therefore, we divide the tool into two main systems, i.e., the social choice system and the deliberation system. These systems can act independently or can be combined to support a decision. The social choice system relies on CSC techniques and allows for the computation of a "socially fair" global ranking of alternatives thanks to the aggregation of elicited individuals' rankings. The deliberation system will provide the argumentation framework which will allow for the computation of justifications on these individuals' rankings. It aims at allowing the "correction" of misinformed or incomplete information by using justifications coming from the different agents. Therefore, with the implementation of these two systems we adapt social choice theory and argumentation in order to have better decisions, in the sense of having justified preferences and their fair collective aggregation. In order to demonstrate the functionality of our tool, we will apply the software procedure on a decision problem extracted from an existing use-case that was conducted for the needs of the Ecobiocap project. The use-case objective is to evaluate the interest of consumers in new-generation packaging. However, note that, this use-case is not restrictive on the applicative usage of the software as we are considering a more general framework for other future possible applications and hence, the tool allows for multiple decision problems.

\section{PROBLEM DESCRIPTION}

We are considering the problem of taking a decision regarding valorization options for agricultural materials with the aid of CSC and AF. Therefore, the problem is formulated as follows. As input, we have a set of alternative options $A$ which will be called alternatives and a set of agents $N$, that will elicit justified preferences on the alternative options. We are considering the case where the agents' preferences are expressed by justified rankings, i.e., each agent provides a total order on the alternatives and a justification for this total order. The collection of the linear orders for all the agents is called the preference profile. In the classical social choice theory, the aggregation of the preferences is computed by a voting rule, i.e., the preference profile is reported to a voting rule (method), which then singles out the winning alternative and the ranking of the remaining ones or a set of winners. 
The agents' preferences and the justifications are used to build the arguments and the argumentation framework $A F$, whose role is to help us elicit the justified preferences.

One should note that an evaluation given by the agent over a set of criteria for the alternatives can serve as justifications for the agent's total order. Taking that into consideration we can see that criteria-based justification can serve as a special case of the multi-criteria decision-making problem.

\section{GENERAL ARCHITECTURE OF THE DECISION SUPPORT SOFTWARE}

In this section we are presenting the general architecture of the decision support software. Our proposal includes the design and the implementation of a decision-making tool, which is split into two main systems, i.e., the social choice system and the deliberation system. Each one of the systems can act as a stand-alone decision tool but they can also be linked together in order to provide a decision. Our tool is composed of the following four subsystems: the data collecting, the voting, the argumentation and the decision subsystems.

\subsection{Data Collecting Subsystem}

The task of this subsystem is to yield the data needed as an input for the voting subsystem and the argumentation subsystem. The most common way for agents to reveal their preferences is through surveys. Hence, this subsystem takes as input the survey's "raw" data, i.e., the preferences of the agents in an abstract format, as well as the justifications behind these preferences. Note that we are considering different use-cases which have different forms of surveys and therefore this subsystem is survey-oriented and the method used to compute the output is adapted each time to the survey format. The output of the subsystem is structured information which contains a total order (ranking) of the alternatives for each agent with potential justifications supporting each preference.

\subsection{Voting Subsystem}

This subsystem's function is to provide methods for electing the "best" alternatives (and/or criteria). Using the term "best", we mean the alternatives/criteria that correspond to the socially fairest outcome and that best reflect the preferences of the agents. To achieve that, we consider a voting setting where we fairly aggregate the set of agents' preferences and produce a ranking of the alternatives or a fixedsized set of equally winning alternatives. We refer to CSC techniques, e.g., voting rules, in order to aggregate the agents' individual preferences. Many voting rules have been proposed in the social choice literature which try to satisfy prominent and fundamental fairness criteria. However, due to the impossibility results by Arrow (1950) and Gibbard-Satterthwaite (Gibbard, 1973; Satterthwaite, 1975) there is no hope of finding a voting rule that can be "perfect", because due to these results some vital criteria can not be satisfied all at the same time. Hence, we propose various voting rules so that the decision maker will be able to select among them.

In social choice literature the single-winner voting rules can be categorized into the scoring methods, e.g., Borda, which are based on scoring protocols and the Condorcet consistent rules, e.g., Copeland, which are based on pairwise comparisons. Condorcet is considered as the founding father of social choice theory and proposed the following rule. An alternative $x$ is said to beat alternative $y$ in a pairwise comparison if the majority of the agents prefer $x$ to $y$, i.e., rank $x$ above $y$. An alternative that beats every other alternative in a pairwise comparison is the Condorcet winner.

The social's choice system projected architecture allows the decision maker to choose if she wants the outcome to be a full ranking of the alternatives or a set of "best" alternatives. Using a classic voting method can handle only the case of aggregating the individual preferences to a full ranking but does not cover the case of finding the set of equally winning alternatives, i.e., the "best" alternatives. Hence, we refer to multi-winner voting rules that permit the fair aggregation of the individual preferences when selecting committees. A committee is a fixed-size subset of alternatives who are equally winners. The goal of these rules is that every agent is represented by a committee 
member and thus these methods are appropriate for electing the best $k$ alternatives, when $k$ is the number of alternatives in the committee. Contrary to the data collecting subsystem which is specific for each survey, the voting subsystem is generic tool which can be used for all the use-cases.

\subsection{Argumentation Subsystem}

The role of this subsystem is to provide the set of coherent and complete points of view. In order to achieve that we use the justifications of the rankings on alternatives/criteria of the different agents and eliminate through logical argumentation the inconsistencies between the agents' preferences. Hence, a deliberation phase interacts with the voting and the ambiguous preferences are eliminated from the preferences of the agents. Following this procedure, we build the justified preference profile, which can serve as input to the voting subsystem.

\subsection{Decision Subsystem}

The role of this subsystem is to provide a recommendation for the decision maker on the given decision problem which is the final output of our software. The recommendation we provide is the parameterized (by the decision maker) outcome of the voting and/or the argumentation subsystem. Figure 1 shows the architecture of the decision support software in high level.

\section{SOCIAL CHOICE SYSTEM}

The social choice system is one of the two systems that compose the decision-making tool and can act independently or in combination with the deliberation system. The system's role is to provide a recommendation that reflects the socially fairest solution. The system provides a quantitative approach and selects the best alternatives with regard to the aggregation of the individual preferences, but if the decision maker demands a more qualitative approach then she has to take into account the deliberation phase, hence the design of the deliberation system which complements this one. The social choice system is composed of an implementation of the following three subsystems: the data collecting, the voting and the decision subsystems. The general architecture of our design defines that the communication between the data collecting and voting subsystems is based on files since a structured json file is the input for the voting subsystem. On the contrary, the voting and decision subsystems communicate internally between the Java code. In this section we present our implementation and

Figure 1. The architecture of the decision support software in high-level

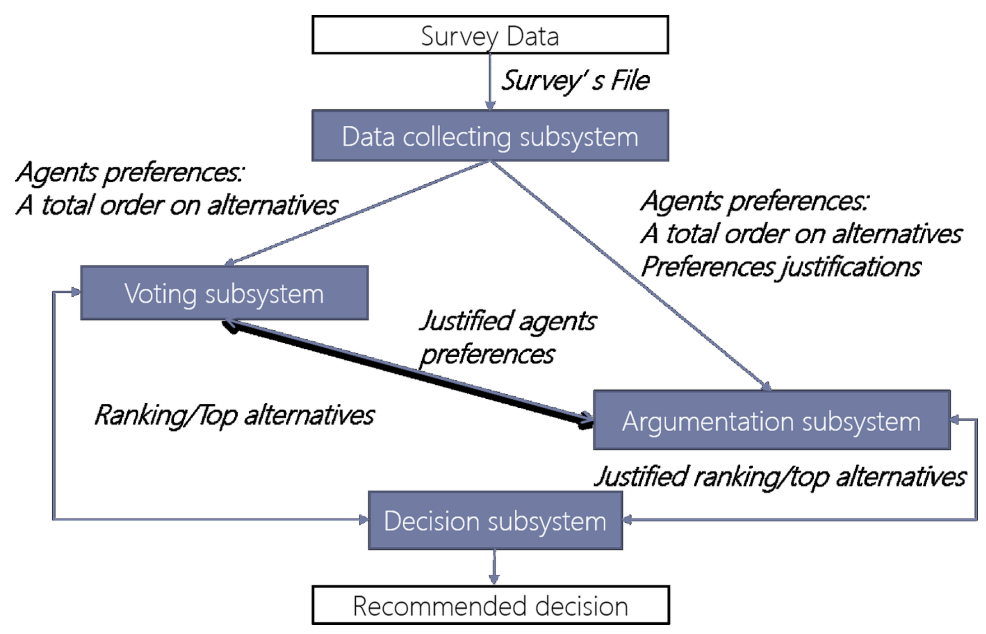


the functionality of the social choice system. The tool is implemented in Java and we use the Swing toolkit for creating the graphical interface.

\subsection{Implementation of the Data Collecting Subsystem}

The role of the data collecting subsystem is to "transform" the raw data which is extracted from different surveys into the suitable input's format for the voting subsystem, i.e., structured information for each one of the questions/decision problems of the survey. Recall that each survey provides us data which is related to the preferences of the agents in an abstract format. Therefore, the mandatory structured information includes the list of the alternatives and the agents' rankings on them. In order to fix the structured information, we construct a structured "json" file format with specific attributes that reflects the format of the survey and hence the voting subsystem can process this file as input. Remember that raw data can have different formats depending on the format of the surveys. Hence, we implement in our code several functions that can handle several different export formats of raw data coming from LimeSurvey (LimeSurvey $\mathrm{GmbH}$, 2003). Their functionality is to transform the different formats into a unique structured data format, and hence to the structured json file. We are implementing the transformation from LimeSurvey, since it is a popular web server-based software which enables users to develop and publish on-line surveys and collect responses, using a userfriendly web interface. The structured json file is designed in a way to correspond to the structured data of the input of the voting subsystem and includes all the information of the surveys. To this end, we standardize the format of the input of the voting subsystem (and hence the json file) in case of future addition of new data input. The structured form of the voting input permits the implementation of additional functions transforming from different surveys formats to the json file. Therefore, the design and the implementation of the data collecting subsystem allows the social choice system to have the capability of including new input formats as long as classes and functions are implemented that can handle the transformation of different file formats to the json standardized input. We have also implemented a direct communication between the data collecting and voting subsystems. This extra feature is directly implemented in the data collecting subsystem and the transformation from the csv file to the voting's subsystem input is done internally. One should note that this is not our general approach but is included in order to facilitate the user and process directly the LimeSurvey's csv file. Figure 2 shows the activity diagram of the data collecting subsystem.

\subsection{Implementation of the Voting Subsystem}

The basic functionality of the voting subsystem is to aggregate agents' preferences and compute the socially fairest alternatives. To achieve that we create a Java class which implements the subsystem's features. We describe them in the reminder of this section.

\subsubsection{Input Type}

There are two input types for the voting subsystem. The default file input's format is a structured json file containing information about the different decision problems and the agents' individual preferences. A single json file can support more than one decision problem. A decision problem is defined by the topic the decision maker designates. Hence, each decision problem corresponds to a question that defines a poll. Therefore, we implement the json file to include several decision problems when multiple questions on the same set of agents are included in the survey. For each question we include in the json file the following information:

- "Question id": A unique number for identifying between the different decision problems.

- "Poll's Question": Contains the formulation of the decision problem in an interrogative form as it is imposed by the decision maker.

- "Alternatives": The list of the alternatives that correspond to the specific decision problem. 
Figure 2. The activity diagram of the data collecting subsystem

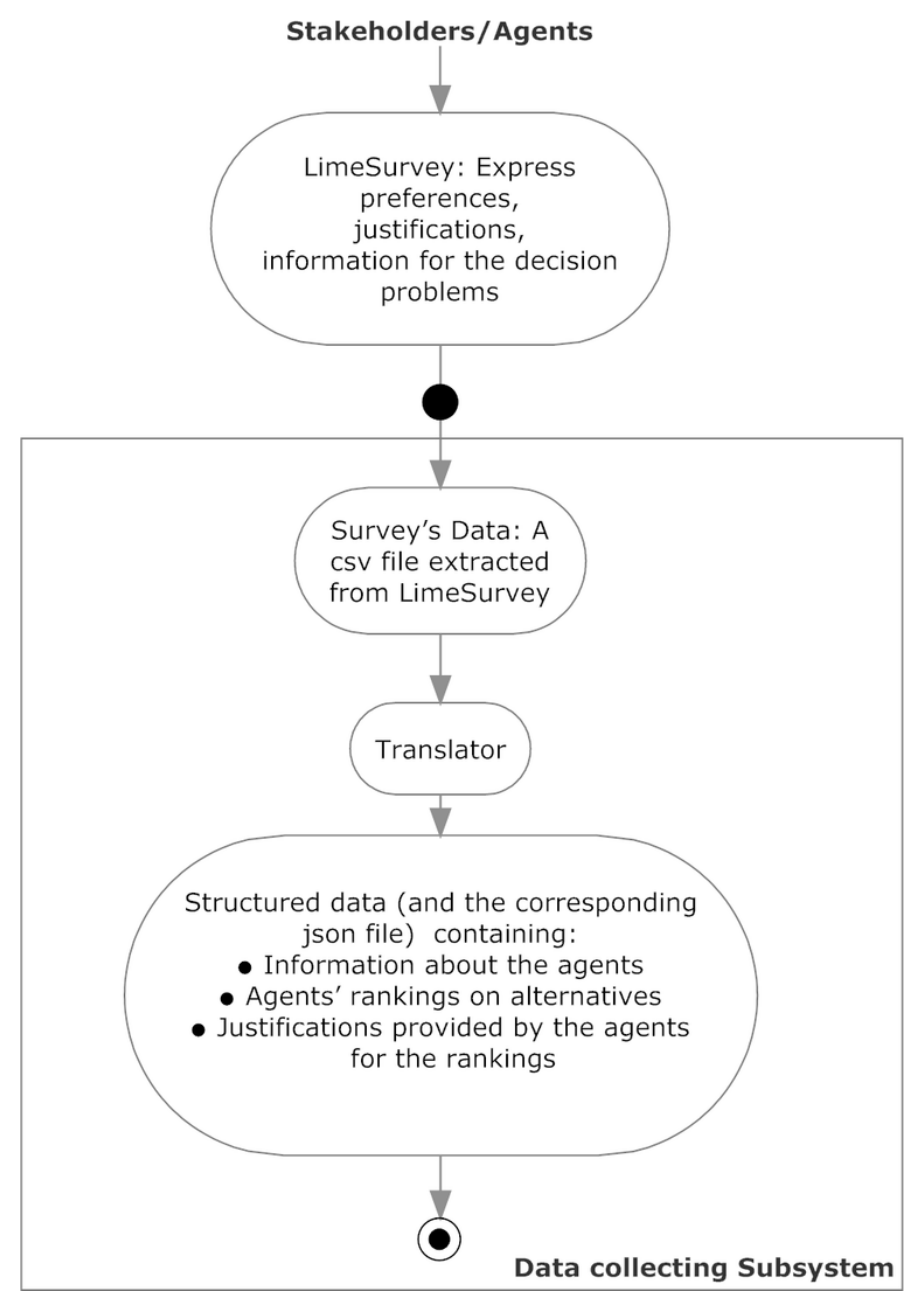

- "Agents characteristics": Contains the categories on which the agents can be classified to. The set of agents can be classified into different categories according to their characteristics. In this field the decision maker defines all the characteristics that she wants to be taken into account for classifying the agents. E.g., one category can be "age".

- "Categories of agents": Contains the sub-categories of the above categories on which the agents can be classified to, e.g., for category "age", we can have the following sub-categories: "1825 ","26-35","36-49","> 50".

For each agent the following information is stored in the json file:

- "Agent id": A number for identifying between the different agents.

- "Values for agents characteristics for Question id $x$ ": Contains the personal information (values) for each agent corresponding to the categories defined in the decision problem $x$.

- "Alternatives ranking for question id $x$ ": Contains the total order of the alternatives according to the agent for problem $x$. 
- "Justification for alternatives ranking for question id $x$ ": Contains the reasoning behind the individual preferences of the agent over the alternatives for problem $x$.

The second input file format is a csv file derived from LimeSurvey containing the above same information in different format. The first line of the csv file is composed of the information about the decision problems and then there is a line for each agent which represents the preferences and the information of this agent.

\subsubsection{Aggregation of Individual Preferences}

The core functionality of the voting subsystem is the aggregation of the agents' individual preferences to a collective preference. Our software proposes to compute the output aggregated ranking using the following voting rules keeping in mind to cover the whole spectrum of the literature: Borda, KemenyYoung, k-approval, Extended Tideman's simplified Dodgson and Multi-winner STV. Therefore, we implement Borda rule (Borda, 1781) as an example of a scoring rule and the Copeland's method (Copeland, 1951) as a representative of Condorcet-consistent rules. We also implement $k$-Approval (Brams \& Fishburn, 1978) because we want to cover the case where the preferences of the agents include a set of equally "accepted" set of alternatives instead of a total order on them. We also implement the Extended Tideman's simplified Dodgson rule (Caragiannis et al., 2014) from the new generation of rules that were deployed recently in the CSC literature to overcome the computational complexity issues of the other rules. This rule has the advantage of being polynomial time and thus can be computed on any input size. In addition, we propose a multi-winner voting rule, i.e., the multi-winner STV, in order to aggregate the agents' individual preferences to a set of equally winning alternatives. A definition of the implemented rules follows.

- Borda's Count: It is a scoring-based voting rule. It is one of the simplest and most intuitive scoring rules, where each alternative receives from 0 to $m-1$ points from each agent, where $m$ is the total number of alternatives. The protocol is that the alternative which is ranked in the k-th position by agent i receives $m-k$ points from this agent. The total Borda score of each alternative is the accumulation of all the points received from the agents. The alternative with the highest accumulated score wins and the rest of the alternatives are ranked according to their score.

- $\quad$ k-Approval: Each agent "approves" (i.e., selects) $k$ out of $m$ alternatives and each one of them gets 1 point. The approval score of an alternative then is the cumulative number of the points she receives from all the agents. In all approval rules (including plurality and veto) the alternative with the highest score wins and the rest of the alternatives follow according to their score. For $k=1$ we obtain the Plurality rule, which is one of the simplest and most fundamental rules in the history of Social Choice. Also, for $k=m-1$ we obtain Veto where each agent approves all but one alternative. The intuition is that each agent poses her disapproval (veto) on one alternative.

- Copeland: In Copeland's rule alternatives are compared pairwise. When an alternative is preferred by the majority of the agents she receives one point and the other alternative receives zero points. In case of a tie, in the classical version of Copeland's rule both receive 0.5 points. The sum over the points is called the Copeland score. Winner(s) are the alternatives with maximum Copeland score and the rest follow according to their score.

- Extended Tideman's Simplified Dodgson Rule: This rule depends also on pairwise comparisons between alternatives and is based on the well-known voting rule of Dodgson (Black, 1958). Since Dodgson's rule is hard to compute we choose this rule because it is a Dodgson's approximation algorithm which can be efficiently computed. This rule is an extension of Tideman's rule and the score of each alternative $x$ is defined as follows. If an alternative is a winner under the Condorcet method then her score is 0 , otherwise her score is computed by the following formula: 
$\operatorname{sc}(x)=m \cdot \operatorname{sc}_{\mathrm{td}}(x)+m(\log m+1)$,

where $m$ is the total number of the alternatives and

$\mathrm{sc}_{\mathrm{td}}(x)=\sum_{y \in \text { alts } .\{\{x\}} \max \{0, k-l\}$

where $k$ is the number of agents that prefer $y$ over $x$ and $l$ is the number of agents that prefer $x$ over $y$. The alternative with the minimum score wins and the rest of the alternatives are ranked according to their score.

- Multi-Winner STV: This rule belongs to multi-winner voting rules which are designed for selecting a fixed $k$-sized committee of alternatives, i.e., the set of $k$-winning alternatives. Hence, multi-winner STV is the most appropriate rule to be used when the output selection is set to $k$-top alternatives. The Single Transferable Vote rule (STV) executes a series of iterations, until it finds $k$ winners. A single iteration operates as follows: If there is at least one candidate with Plurality score at least $\mathrm{q}=\lfloor\mathrm{n} /(\mathrm{k}+1)\rfloor+1$, then an alternative with the highest Plurality score is added to the committee; then $q$ voters that rank her first are removed from the election (a randomized tiebreaking plays an important role here), and the selected alternative is removed from all voters' preference orders. If there is no such alternative, then an alternative with the lowest Plurality score is removed from the election (again, ties are broken uniformly at random). The Plurality scores are then recomputed.

As noted previously, there is no "best" voting rule and on the same input, i.e., the preference profile of the agents, different voting rules can compute different aggregated rankings as output, leading to the need, for the decision maker, to be able to choose the voting rule used for computation. In the following example we are showing that phenomenon.

- Example. Let us consider the following individual preferences for the agents. We have 55 agents with the following rankings. We should note here that $\mathrm{x}>\mathrm{y}$ indicates that an agent prefers $\mathrm{x}$ to $\mathrm{y}$.

- 18 agents: $\mathrm{A}>\mathrm{D}>\mathrm{E}>\mathrm{C}>\mathrm{B}$

- 12 agents: $\mathrm{B}>\mathrm{E}>\mathrm{D}>\mathrm{C}>\mathrm{A}$

- 10 agents: $\mathrm{C}>\mathrm{B}>\mathrm{E}>\mathrm{D}>\mathrm{A}$

- 9 agents: $\mathrm{D}>\mathrm{C}>\mathrm{E}>\mathrm{B}>\mathrm{A}$

- 4 agents: $\mathrm{E}>\mathrm{B}>\mathrm{D}>\mathrm{C}>\mathrm{A}$

- 2 agents: $\mathrm{E}>\mathrm{C}>\mathrm{D}>\mathrm{B}>\mathrm{A}$

We are now applying the following different voting rules and obtain the corresponding results.

- Borda: Alternative D wins with $(18 \times 3)+(12 \times 2)+(10 \times 1)+(9 \times 4)+(4 \times 2)+(2 \times 2)=136$ points. A gets 72, B 101, C 107 and E 134.

- $\quad$ 1-Approval (Plurality): Here, A wins, with 18 votes. Alternatives B to E have score 12, 10, 9 and 6 respectively.

- For Tideman's and Copeland's rules we observe that a Condorcet winner exists so she is also the winner under these rules. Alternative $\mathrm{E}$ is the Condorcet winner since she beats each of the other four options in pairwise comparisons, i.e., E beats A 37-to-18, B 33-to-22, C 36-to-19, and D 28-to-27. 
- For Multi-Winner STV suppose that we want the best 3 alternatives, so we fix $k=3$. A has plurality score of 18 which is at least $[55 /(3+1)\rfloor+1=14$, so $\mathrm{A}$ is included in the winning alternatives and the 18 voters that rank A first are removed from the election, and also A is removed from all voters' preference orders. B has plurality score of 12 which is at least $\lfloor 37 /(3+1)\rfloor+1=10$, so B is also included in the winning alternatives and the 12 voters that rank B first are removed from the election, and also B is removed from all voters' preference orders. C has plurality score of 10 which is at least $\lfloor 25 /(3+1)\rfloor+1=7$, so $\mathrm{C}$ is also included in the winning alternatives. Hence, $\mathrm{A}, \mathrm{B}$ and $\mathrm{C}$ are the winners.

- Incomplete (Truncated) Preferences: It is the case where agents do not provide a complete ranking of the alternatives. This can happen when each agent is likely to know who her most favorite alternatives are but she is unwilling to put the effort into ranking the remaining ones. Then, it is safe to assume that she likes them less than the ranked ones but among the unranked ones there is no difference in preference. We use this assumption when computing the collective preferences under incomplete preferences. Therefore, the typical voting rules, like the abovementioned, have to be adjusted to cover this case. For example, for Borda, we could assume that each unranked alternative receives 0 points from a given vote (a method used in Slovenia, which is called pessimistic scoring model). Another method for Borda is the optimistic scoring model, where if there are $m$ alternatives but a vote ranks only $k$ of them, then the ranked alternatives get $m-1, \ldots, m-k$ points (depending on their position in the ranking) and each unranked alternative gets $m-k-1$ points. We will use the pessimistic model in our computation for Borda because it is more widely used. For $k$-approval if the preferences of the agents are incomplete but more than $\mathrm{k}$ alternatives are ranked then there is no difference and on the contrary when less than $\mathrm{k}$ alternatives are ranked by the agent, then all get one point. For the rules based on the pairwise comparisons we assume that if an alternative is ranked and the other is not, then the winner is the one ranked and when both are unranked we do not take into account this comparison. Figure 3 shows the activity diagram of the voting subsystem and Figure 4 shows the diagram of the decision subsystem.

\subsection{Implementation of the Decision Subsystem}

The objective for the decision subsystem is to provide a recommendation for the decision maker. The output (decision) will be based on the aggregation of the individual preferences but may contain different results according to the parameters that are imposed by the decision maker. To achieve that we create a Java class which implements different features, which we call decision-aiding features. There is a link in the implementation of voting and decision subsystems since decision-aiding and voting features interact. The decision maker can choose amongst decision-aiding features that can be classified into 3 categories according to the computation phase in which they belong to.

\subsubsection{Voting Phase}

The first parameter on which the decision maker can base her decision is the choice of the appropriate voting rule. The decision maker can therefore choose one or more of the abovementioned rule(s) according to her needs and obtain different decisions, since different rules on the same preference profile can lead to different collective rankings.

\subsubsection{Aggregation Phase}

During the decision-making procedure choosing the right voting rule is not the only crucial matter in order to support a decision. The decision maker is provided with different aggregation procedures that can help her take a better decision according to the needs of the problem by either taking a subset of the agents preferences or/and increasing the weight of the opinion of some agents. The preference profile can be partitioned in different subsets when agents share common characteristics. When agents belong to the same subset we say that they belong to a specific category, e.g., agents can be 
Figure 3. Activity diagram of the voting subsystem

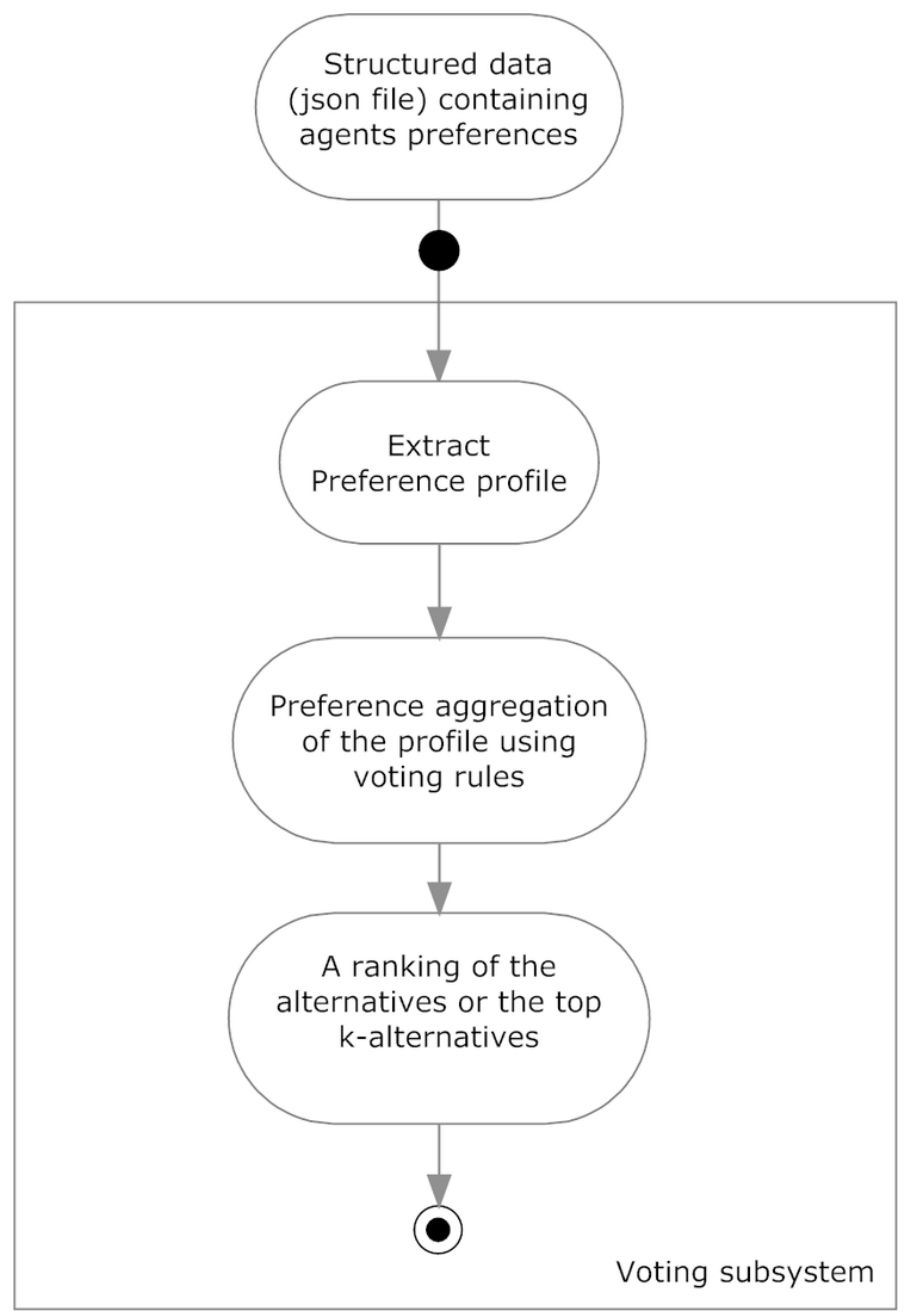

partitioned according to sex and hence we have two categories, i.e., male and female. It is therefore the preference profile which is taken as the input the one that differentiates the aggregation procedures. The decision maker can also combine categories in order to get the preference profile for the input (combination of categories). E.g., the decision maker can choose her input to be men aged 18-35. To get the desired input, the software allows the option to combine categories "Sex: male" and "Age: 18-35". Therefore, we propose the following decision-aiding aggregation procedures.

- Total Aggregation: The decision is computed taking into account all agents' preferences.

- Aggregation per category: In this procedure the agents are forming groups (subsets) according to their classification on different categories. Therefore, we apply a voting rule having as input the preference profile that corresponds to agents belonging only on the specified, by the decision maker, category (or combination of categories).

- 2-Phase Aggregation: Also, in this procedure the agents are forming groups according to their category. In the first phase a ranking for each one of the agents' categories (subsets) is computed by applying a voting rule. In the second phase the decision is the collective preference computed 


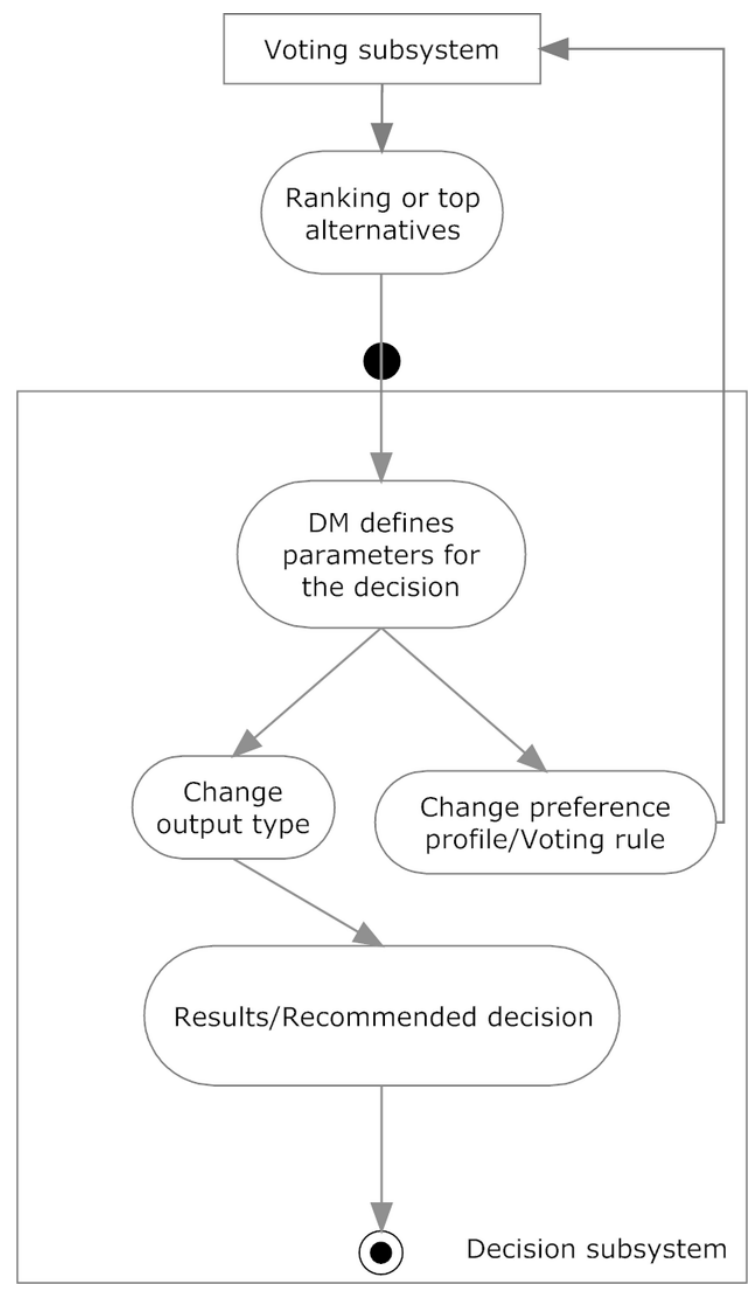

by the aggregation of the rankings produced during the first phase. For both steps we are using the same rule. We consider that each one of the agents' types has equal say (i.e., weight) to the collective preference.

- 2-Phase Aggregation with Weights: It is similar to the above procedure but in this case we do not consider that each one of the agents' types has equal say to the final collective preference. Instead we provide the decision-maker the ability to define the weights for the different categories according to the needs of the decision problem. The final ranking is computed by the proportional, according to the weights, aggregation of the votes of each category.

\subsubsection{Output Phase}

During this phase the decision maker can base her decision on different types of output. The output produced by the tool can have the following forms.

- Single Winner: The recommendation that is provided to the decision maker is the winning alternative according to the specified rule. In case of a tie we can have multiple winners. 
- $\quad$ k-Top Alternatives (Committee Size): The recommendation is a committee of size $k$, which corresponds to the set of the best $\mathrm{k}$ alternatives. A multi-winner voting rule is used.

- Rank: The recommendation is provided in the form of a complete ranking over the alternatives.

\subsection{Graphical User Interface}

The goal of our decision support software is to be used by decision-makers which are non-voting experts. Taking this goal into account, we implement a graphical user interface (GUI) which corresponds to the functionalities described in the subsystems. We believe that the GUI is user-friendly and permits the decision maker to explore all the functionalities in terms of preference aggregation and achieve a decision based on her needs. A screenshot of the GUI is shown in Figure 5.

The usage of the tool can be seen through the layout of the interface which is the following. On the upper part of the application we depict the data collecting subsystem where the user can load the survey's file (csv) or the voting input file (json). Right beneath is the translator where one can transform the survey csv file into the json file format which is appropriate for input to the voting subsystem. In the middle part of the GUI the voting subsystem is being visualized where the decision maker can choose the voting rule that is going to be used for the aggregation of the preference profile.

Figure 5. A screenshot of the GUI

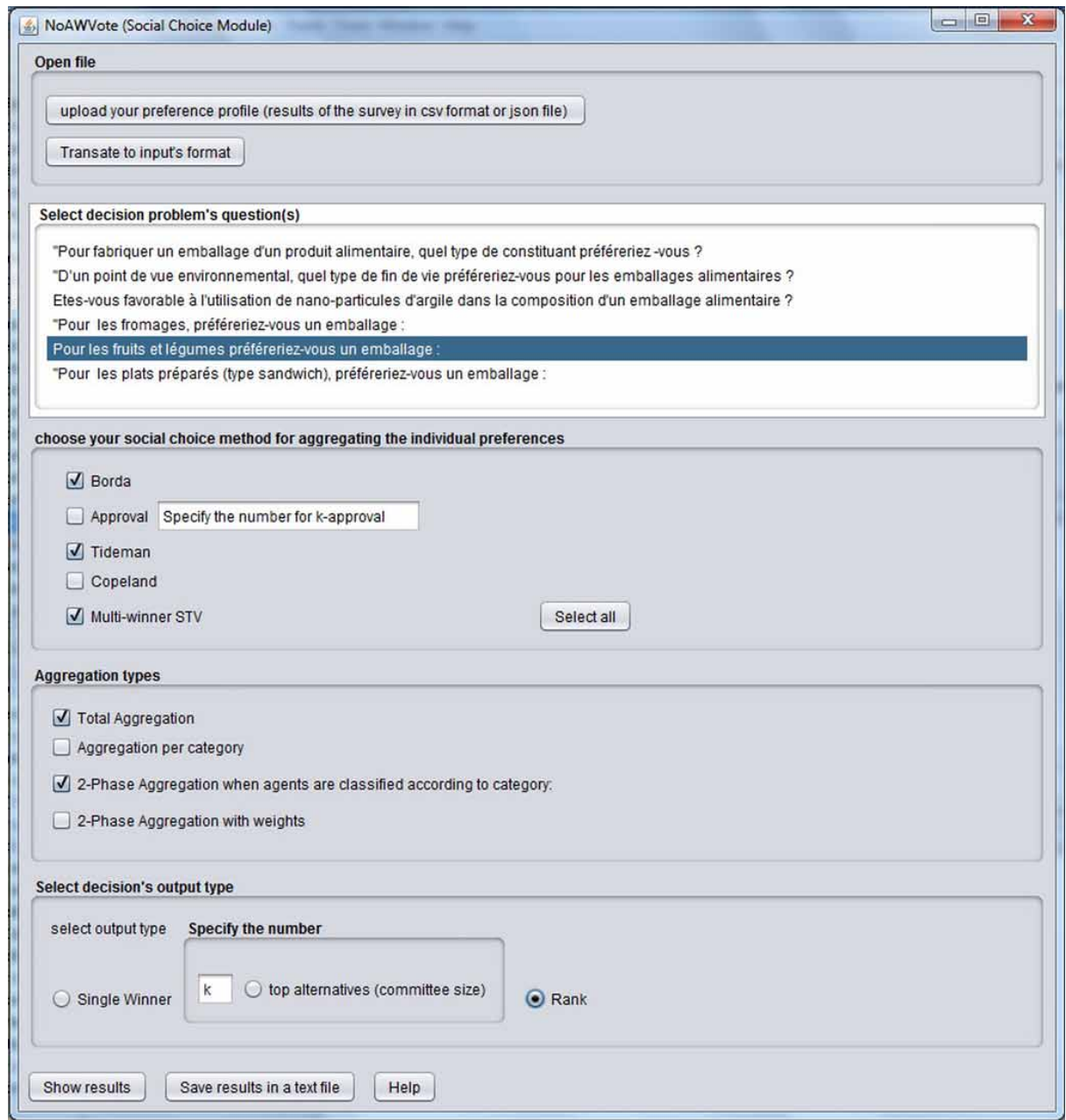


The decision maker has the option to choose among different rules and may obtain different results. Then in the lower two panels the decision subsystem is being shown, where the decision maker can choose the aggregation and output types. In order to select the aggregation types a new window appears (Figure 6). In this example the decision maker takes into account only the agents aged 26-35.

The output of the computation is depicted in a text form. The decision maker can choose through the interface the parameters she wants to impose and hence obtain the corresponding results. The subpart of the interface for the results is depicted in Figure 7. We also provide the option to save the results in a text file.

\section{EXAMPLES ON REAL DATA: PACKAGING CHOICE DECISIONS FOR ECOBIOCAP AND OPINION ANALYSIS}

In order to demonstrate the usability of our tool and validate the presented methodology we study real-data decision problems on food packaging selection. The studied decision problem aims at recommending relevant alternate food packaging based on multiple criteria. One critical factor in recommending new packaging is the opinion of the consumers. Therefore, we apply our tool to this problem and obtain the preferences of the agents from a case study which is extracted from a survey that was conducted in a national level for France for the needs of the Ecobiocap (ECOefficient BIOdegradable Composite Advanced Packaging) project. This survey is related to different functionalities regarding food packaging where 235 citizens helped by answering the questions. Therefore, we use this survey for collecting the data, i.e., the citizens (agents) preferences and the justifications, and answering in the next step the decision problem. One should observe that using our applied procedure on these examples is not restrictive on the applicative usage of our software since it is designed as a more general framework which can support future possible applications and problems.

Figure 6. Interface for aggregation types

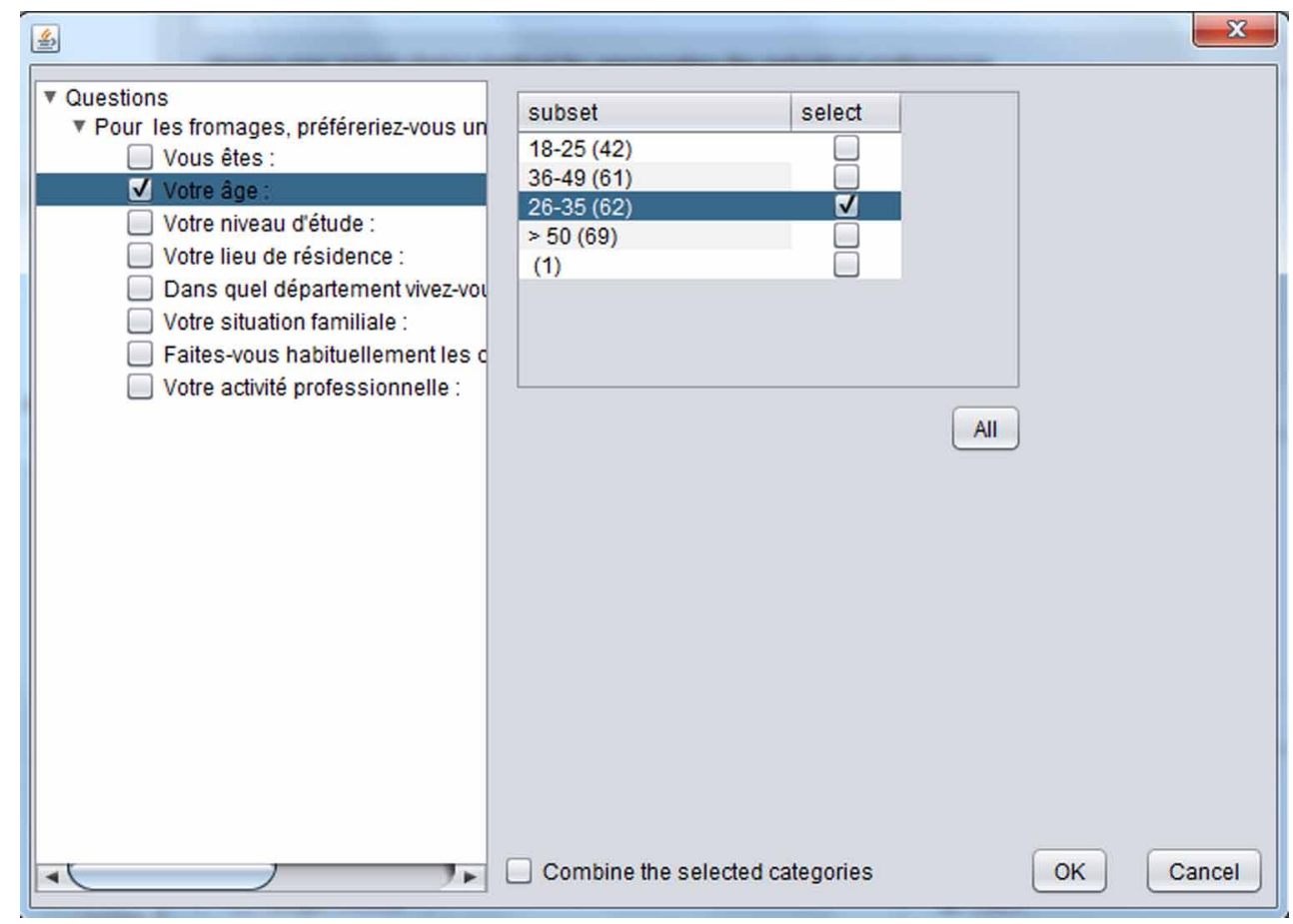




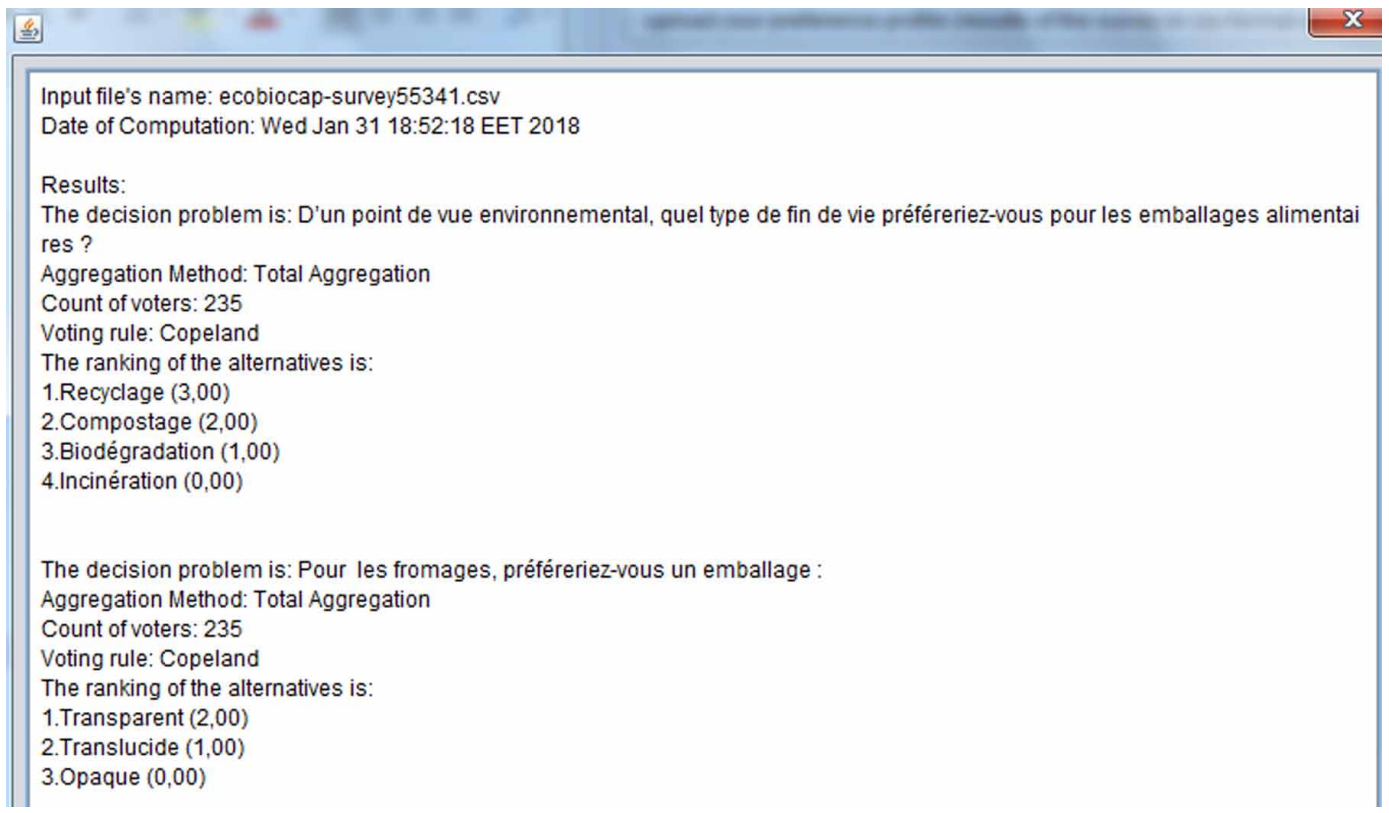

The first category of problems included in this survey consists in finding the best alternatives for food packaging on different kinds of foods. Hence, the citizens were asked to give their preferences on different questions regarding cheese, fruits \& vegetables and sandwich packaging. These questions correspond to different decision problems (for each type of food) as the agents evaluated and ranked the alternatives for each question separately. The alternative options given to be ranked by each agent were transparent, translucent and opaque.

The second decision problem on which the agents were asked to give their opinion concerns the type of the material for food packaging. Therefore, each agent gave her preference on the type of the material she prefers for producing the food packaging. The agents had to choose between two alternatives for this problem, i.e., bio-source (in particular, using agricultural by-products to produce the packaging) and petro-source.

For the next decision problem each agent had to answer if she approves the usage of clay nanoparticles for the composition of the food packaging. The agents had to choose between yes and no.

The last decision problem is related to the end-of-life management of the food packaging. The agents gave their preferences on the type of the end-of-life they prefer for the packagings. For this problem the set of alternatives were: recycling, composting, biodegradation and incineration. Figure 8 shows multi-criteria decision support results interface.

\subsection{Preference Aggregation for Ecobiocap's MCDSS}

In this application, the social choice system computed aggregated preferences associated with different populations for specific food packaging characteristics. Then, those aggregated preferences have been used as input parameters for a multi-criteria decision support system - called MCDSS (Destercke et al., 2011; Guillard et al., 2015) which retrieves the most relevant packaging for a given food. In the following, we present the results obtained for cheese packaging regarding transparency. Using individual preferences expressed by 235 citizens in the Ecobiocap survey, our tool has computed the following aggregated preferences: 
Figure 8. Multi-criteria decision support (MCDSS) results interface

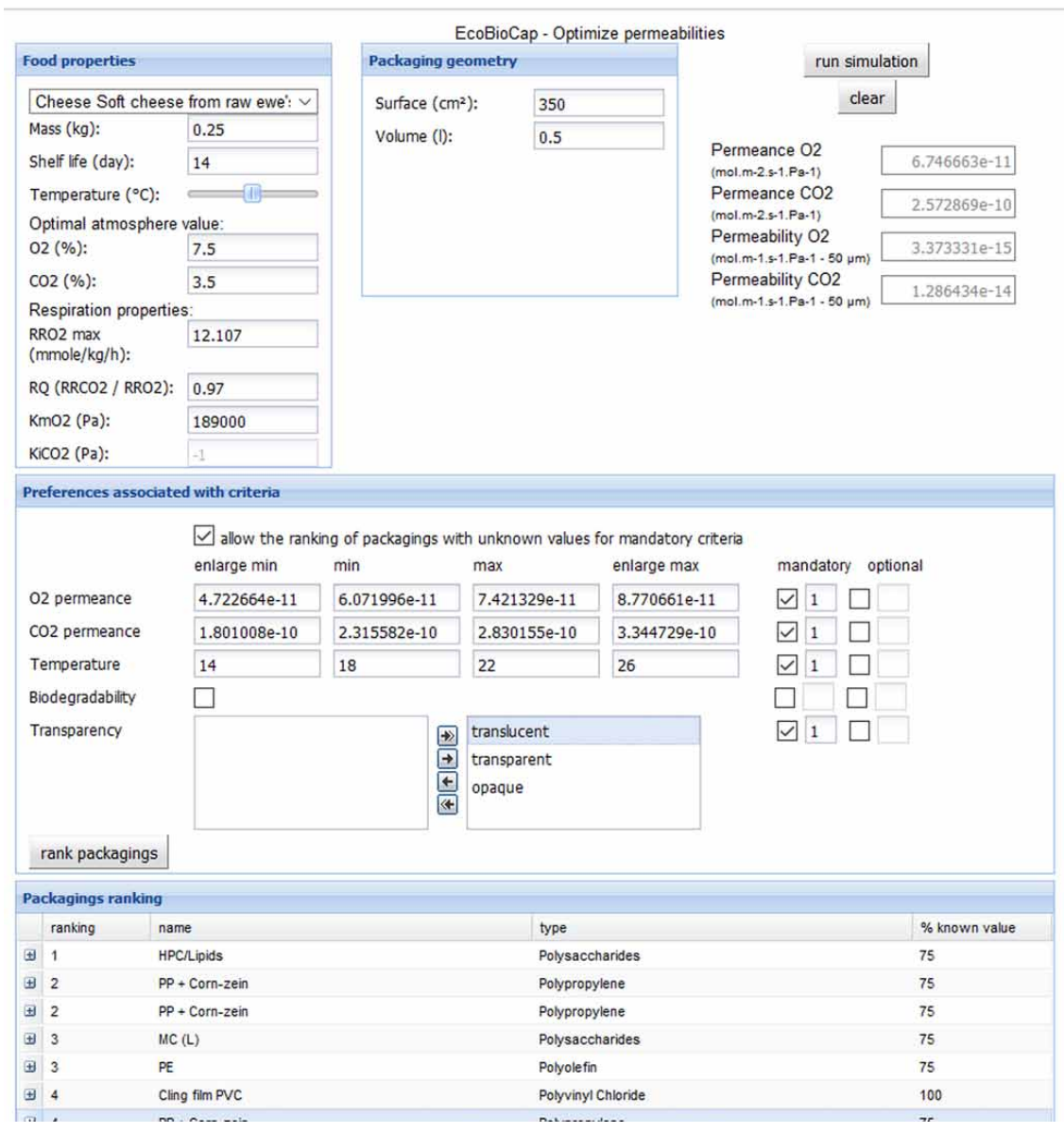

- Case 1: French population as a whole (235 votes): transparent $>$ translucent $>$ opaque

- Case 2: people living in Ile de France (46 votes): translucent $>$ transparent $>$ opaque

The MCDSS has been used twice to retrieve the most relevant packaging to pack a soft cheese from raw ewe's milk (250 grams, shelf life of 14 days, storage at ambient temperature) using preferences associated with populations of cases 1 and 2 . For Case 1, the best packaging is a MC(L) Polysaccharide as for Case 2, the best one is a HPC/Lipids Polysaccharide. The complete list of most preferred packaging for Case 2 is presented in the "Packaging ranking" window in the lower part of Figure 8 . Those results have been presented to stakeholders of the food packaging chain during the CIAG conference (Buche et al., 2017).

\subsection{Opinion Analysis as A Powerful Tool for Decision-Making}

Another possible use of the system is to provide recommendations to the decision maker for designing potential food packagings taking into account the citizens opinion.

The results revealed that for the type of packaging the citizens prefer for cheese, fruits \& vegetables and sandwich the transparent is ranked higher than translucent while opaque is the last one in all but the plurality rule. In the case of 2 or more alternatives plurality is not considered an appropriate 
method by social choice theorists. Hence, the recommendation provided to the decision maker is than citizens prefer transparent to translucent to opaque.

Regarding the second decision problem, the results revealed that in the collective preference of the citizens for the type of the material they prefer for producing the food packaging the bio-source is ranked higher than petro-source. The same result appeared when all the voting rules were used for the aggregation of their individual preferences. Hence, the recommendation provided to the decision maker is than citizens prefer bio-source to petro-source.

For the decision problem concerning the usage of clay nanoparticles for the composition of food packaging, we used plurality and the recommendation provided to the decision maker is than citizens do not prefer the usage of clay nanoparticles.

The results for the last decision problem, which corresponds to the end-of-life for packaging, revealed the following same ranking for the rules of Borda, Tideman's and Copeland: recycling, composting, biodegradation and incineration. Using plurality we obtained a slight different ranking: recycling, biodegradation, composting, and incineration but observe that the winner is still the same under all voting rules. In this case we have four alternatives so we will not use the ranking provided by plurality and hence, the recommendation provided to the decision maker is than citizens prefer recycling to composting to biodegradation to incineration for the end-of-life for food packaging.

Summing up the results of all the above decision problems the decision provided by the collective preferences of the citizens is that they prefer a food packaging that satisfies the following features. It should be transparent, made of bio-source materials without the use of clay nanoparticles and should also be recyclable.

Also, based on the decision maker parameters the following interesting insights appeared when using different voting rules and aggregation types for the aggregation of the individual preferences. The first category of the observed insights in real data examples concerns the discrepancies for the same preference profile when a different voting method was used. For example, as already mentioned the case of plurality compared to the other voting rules. The second category of insights observed are the ones related to the aggregation when different subsets of citizens (i.e., partitions of the preference profile) were taken into account. We make the following interesting observations. Note that all the remarks that we make below refer to computation under the same voting rule.

- Observation 1: For the problem of the end-of-life, the collective ranking according to different sex is different when men are compared to women. The collective preference of the 91 men included in the survey is: recycling, biodegradation, composting and incineration. On the other hand, 143 women voted for the survey and their collective ranking is: composting, recycling, biodegradation and incineration.

- Observation 2: For the same problem different results are also obtained if we change the category of aggregation to age. All the age groups except the ones over 50, i.e., "18-25", "26-35", "36-49", have the same ranking: recycling, composting, biodegradation and incineration while people over 50 have composting, biodegradation, recycling and incineration.

- Observation 3: The end-of-life problem is not the only one with interesting insights. For the problem of cheese packaging we also obtain different rankings when different subsets of the preference profile are used. For example, citizens living in the Herault department have the following preference: transparent, translucent, opaque while people living in Paris have: translucent, transparent, opaque.

Therefore, we can see in practice that collective preferences change according to a partition of the preference profile used as the input. Therefore, we provide the partitioning of the preference profile as an important tool to the decision maker in order to support her decision when the nature of the decision problem is driven by different constraints. For example, if the decision maker is only interested for producing a cheese packaging that will only be sold in Paris then she should rely her decision only 
on the preferences of the agents that live in Paris (observation 3). We believe also that the 2-phase aggregation is a really important tool for the decision maker as we state in the next observation.

- Observation 4: For the end-of-life problem we can see that people living in Herault have different preferences from people living in Paris. People from Herault prefer recycling $>$ composting $>$ biodegradation $>$ incineration while people from Paris equally prefer composting and biodegradation to recycling and incineration. The total of aggregation of the all the agents produces the collective ranking: recycling $>$ composting $>$ biodegradation $>$ incineration which is the same as the Herault's collective ranking. This is due to the ratio of the statistical sample which is not proportional to the real population ratio because in the survey we had 137 agents voting from Herault and 14 from Paris. However, if the decision maker wants a fairest solution according to the population ratio she can adjust the weights of the voters living in different regions. Hence, if we assume weight of 12 to Paris compared to 1 for Herault (12 million inhabitants versus 1 million) then the following collective ranking is computed: composting > biodegradation $>$ recycling $>$ incineration. Note that, we also assign zero weight to the other departments since the statistical sample is small for them.

\section{CONCLUSION AND FUTURE WORK}

In this paper, we have proposed a software tool for decision-making for issues coming from agricultural engineering with the aid of Computational Social Choice and Argumentation Framework. We describe thoroughly the social choice system of the decision support software and demonstrated its application on a real-data example in the context of Ecobiocap project where the objective is to find the best food packaging in terms of material, visibility and end-of-life.

As future work, we want to further extend our research towards the integration of Argumentation and Social Choice for "better" decisions. Hence, our goal is to design and implement the deliberation system which will complete the decision support software.

\section{ACKNOWLEDGMENT}

We thank Madalina Croitoru for her contribution in the general design of the decision tool and for her help in drawing Figure 1. The research leading to these results is part of NoAW project that has received funding from the European Union's Horizon 2020 research and innovation programme under grant agreement No 688338. The research was partially implemented with a scholarship from IKY funded by the action "Support of Postdoctoral Researchers" from the resources of the EP "Human Resources Development, Education and Lifelong Learning" with priority axes 6, 8, 9 and is and cofunded by the European Social Fund - ESF and the Greek state. 


\section{REFERENCES}

Amgoud, L., Bonnefon, J. F., \& Prade, H. (2005). An argumentation-based approach to multiple criteria decision. In European Conference on Symbolic and Quantitative Approaches to Reasoning and Uncertainty (pp. 269-280). Springer Berlin Heidelberg. doi:10.1007/11518655_24

Amgoud, L., \& Prade, H. (2009). 'Using arguments for making and explaining decisions'. Artificial Intelligence, 173(3-4), 413-436. doi:10.1016/j.artint.2008.11.006

Arrow, K., \& Raynaud, H. (1986). Social choice and multicriterion decision making. Cambridge: MIT Press.

Arrow, K. J. (1950). A difficulty in the concept of social welfare. Journal of Political Economy, 58(4), 328-346. doi:10.1086/256963

Benayoun, R., Roy, B., \& Sussman, B. (1966). ELECTRE: une méthode pour guider le choix en présence des points de vue multiples (technical report). SEMA-METRA International.

Black, D. (1958). Theory of Committees and Elections. Cambridge University Press.

Bonnefon, J. F., \& Fargier, H. (2006). Comparing sets of positive and negative arguments: Empirical assessment of seven qualitative rules. Frontiers In Artificial Intelligence And Applications, 141, 16.

Borda, J.-C. d. (1781). Mémoire sur les élections au scrutin. Histoire de l'Academie Royale des Sciences.

Bouveret, S. (2010). Whale ${ }^{4}$ Which alternative is elected. Retrieved from http://strokes.imag.fr/whale4

Brams, S. J., \& Fishburn, P. C. (1978). Approval voting. American Political Science Review, 72(3):831-847, 009.

Brandt, F., Chabin, G., \& Geist, C. (2015). Pnyx: A Powerful and User-friendly Tool for Preference Aggregation. In Proceedings of the 2015 International Conference on Autonomous Agents and Multiagent Systems (pp. $1915-$ 1916). International Foundation for Autonomous Agents and Multiagent Systems.

Buche, P., Gontard, N., Guillard, V., Guillaume, C., \& Menut, L. (2017). Outil d'aide à la sélection d'emballages alimentaires pour la conservation sous atmosphère modifiée des produits frais. Innovations Agronomiques, 58, 81-91. Retrieved from https://prodinra.inra.fr/record/416054

Caragiannis, I., Kaklamanis, C., Karanikolas, N., \& Procaccia, A. D. (2014). Socially desirable approximations for Dodgson's voting rule. ACM Transactions on Algorithms, 10(2), 1-28. doi:10.1145/2556950

Copeland, A. (1951). A "reasonable" social welfare function. Seminar on applications of mathematics to social sciences.

Destercke, S., Buche, P., \& Guillard, V. (2011). A flexible bipolar querying approach with imprecise data and guaranteed results. Fuzzy Sets and Systems, 169(1), 51-64. doi:10.1016/j.fss.2010.12.014

Fox, J., \& Parsons, S. (1997). On using arguments for reasoning about actions and values. In Proceedings of the AAAI Spring Symposium on Qualitative Preferences in Deliberation and Practical Reasoning (pp. 55-63). AAAI Press.

Gibbard, A. (1973). Manipulation of voting schemes: A general result. Econometrica, 41(4), 587-601. doi:10.2307/1914083

Guillard, V., Buche, P., Destercke, S., Menut, L., Guillaume, C., \& Gontard, N. (2015). A Decision Support System for designing biodegradable packaging for fresh produce. Computers and Electronics in Agriculture, 111, 131-139. doi:10.1016/j.compag.2014.12.010

Karacapilidis, N. I., \& Papadias, D. (1998). A group decision and negotiation support system for argumentation based reasoning. In Selected Papers from the Workshop on Reasoning with Incomplete and Changing Information and on Inducing Complex Representations (pp. 188-205). doi:10.1007/3-540-64413-X_36

Keeney, R. L., \& Raiffa, H. (1993). Decisions with multiple objectives: preferences and value trade-offs. Cambridge university press. doi:10.1017/CBO9781139174084

LimeSurvey GmbH. (2003). LimeSurvey. Retrieved from https://www.limesurvey.org/

Merlob, B., Procaccia, A. D., Shah, N., \& Wang, P. (2016). http://robovote.org/

Morge, M., \& Mancarella, P. (2007). The hedgehog and the fox. An argumentation-based decision support system. In Proceedings of the 4th International Workshop on Argumentation in Multi-Agent Systems (pp. 55-68), 
Ostanello, A. (1985). Outranking methods. In Multiple Criteria Decision Methods and Applications (pp. 41-60). Springer Berlin Heidelberg. doi:10.1007/978-3-642-70583-0_4

Roy, B. (1968). Classement et choix en présence de points de vue multiples. Revue française d'automatique, d'informatique et de recherche opérationnelle, 2(1), 57-75.

Roy, B. (1991). The outranking approach and the foundations of electre methods. Theory and Decision, 31(1), 49-73. doi:10.1007/BF00134132

Roy, B., \& Bouyssou, D. (1993). Aide multicritère à la décision: Méthodes et cas. Paris: Economica.

Satterthwaite, M. A. (1975). Strategy-proofness and arrow's conditions: Existence and correspondence theorems for voting procedures and social welfare functions. Journal of Economic Theory, 10(2), 187-217. doi:10.1016/0022-0531(75)90050-2

Tamani, N., Mosse, P., Croitoru, M., Buche, P., Guillard, V., Guillaume, C., \& Gontard, N. (2015). 'An argumentation system for eco-efficient packaging material selection'. Computers and Electronics in Agriculture, 113, 174-192. doi:10.1016/j.compag.2015.02.012

Von Winterfeldt, D., \& Edwards, W. (1986). Decision analysis and behavorial research. Cambridge: Cambridge University Press.

Yun, B, Bisquert, P, Buche, P, \& Croitoru, M. (2016). Arguing About End-of-Life of Packagings: Preferences to the Rescue. MTSR: Metadata and Semantics Research, 672, 119-131.

Nikos Karanikolas is currently a post-doctoral researcher in the University of Patras, Greece. He received his PhD degree from the Department of Computer Engineering and Informatics, University of Patras, in 2014. He has been a post-doctoral researcher in the LIG, University of Grenoble-Alpes, France from October 2014 to October 2015 and a post-doctoral researcher in INRA, French Agricultural Research Institute \& LIRMM, University of Montpellier, France from November 2016 to November 2017. His research interests lie in the areas of Computational Social Choice, Information Visualization, Argumentation Systems and Design/Analysis of Algorithms.

Pierre Bisquert received his PhD degree in computer science from the University of Toulouse, France, in 2013. $H e$ is an INRA research scientist in the IATE laboratory and a permanent member of the INRIA GraphIK team. His work focuses on collective decision making with a particular interest in the understanding of rational persuasive and argumentative processes, and in the aggregation of individual preferences through voting rules.

Patrice Buche received the $P h D$ degree in computer science from the University of Rennes, France, in 1990. He has been assistant professor with AgroParisTech, Paris from 1992 to 2002 and a research engineer with INRA, French Agricultural Research Institute since 2002. His research works mainly concern data and knowledge integration from heterogeneous sources, fuzzy querying in structured and weakly structured databases, computational social choice and argumentation for decision support with applied projects in food and biobased product engineering.

Christos Kaklamanis received his S.B. in Computer Science and Engineering from Massachusetts Institute of Technology, Cambridge, MA, USA (1986). Then he obtained his S.M. (1989) and his Ph.D. (1992) in Computer Science from Harvard University, Cambridge, MA, USA. He is Full Professor at the Department of Computer Engineering and Informatics of University of Patras where, from 2009 to 2011 and from 2003 to 2005 he served as Vice-Chair and from 1997 to 2003, he served as Director of the "Division of Applications and Foundations of Computer Science". He is also President of Computer Technology Institute \& Press "Diophantus" (CTI), Patras, Greece, where from 2007 to 2013 he served as Vice President and from 2013 to 2016 he served as Acting President. His research interests include design and analysis of algorithms, computational complexity, communication networks, parallel and distributed computing, randomized and approximation algorithms, algorithmic game theory, computational social choice, high-performance computing, cryptography, ICT in education.

Rallou Thomopoulos, HDR (habilitation to conduct research groups and PhDs), is INRA Research Scientist in Montpellier and permanent member of the GraphIK lab at INRIA. She has dual skills in computer science and the agri-food sector. Her research topics are in knowledge representation and decision support, focused on argumentation systems and complemented by simulation approaches, applied to agri-food systems. She was a Guest Research Scientist at Laval University (Canada) in 2015-2016 and previously Assistant Professor at AgroParisTech. 